\title{
Correction to: Loss of PTEN expression is associated with PI3K pathway-dependent metabolic reprogramming in hepatocellular carcinoma
}

\author{
Chuanzong Zhao ${ }^{1,2}$, Ben Wang ${ }^{1}$, Enyu Liu ${ }^{1}$ and Zongli Zhang ${ }^{1 *}$
}

\section{Correction \\ Cell Communication and Signaling (2020) 18:131 https://doi.org/10.1186/s12964-020-00622-w \\ Following publication of the original article [1], the authors identified the following errors.}

- In the first paragraph of the Results/PTEN is downregulated in HCC and correlates with poor prognosis of HCC section, "tumor classification and metastasis" should be replaced with "tumor size, classification and metastasis".

- In the Results/Overexpression of PTEN inhibits Warburg effect and maintains mitochondrial function in HCC cells section, "a decreased proportion of red to green fluorescence" should be revised to "increased proportion of red to green fluorescence and recovered mitochondrial function".

- In the legend of Fig. 3 "Ratio of red/green fluorescence intensity" should be changed to "Mitochondrial membrane potential $\Delta \Psi^{\prime \prime}$. to: Further to this, the following change have been made to the Figs. 1, 2, 3, and 4.

- Figure 1: A and B, group typesetting name is inverted. The correct sequence is from left to right: adjacent to cancer, liver cancer, and also marked with PTEN and PI3K

- Figure 3: C, vector NC and OE PTEN group image typesetting and statistical errors, I revised the image typesetting

- Figure 4: the statistics of averctor NC and OE PTEN group are wrong. I revised the picture layout again

The updated figures and captions, as well as tables are given below: original author(s) and the source, provide a link to the Creative Commons licence, and indicate if changes were made. The images or other third party material in this article are included in the article's Creative Commons licence, unless indicated otherwise in a credit line to the material. If material is not included in the article's Creative Commons licence and your intended use is not permitted by statutory regulation or exceeds the permitted use, you will need to obtain permission directly from the copyright holder. To view a copy of this licence, visit http://creativecommons.org/licenses/by/4.0/. The Creative Commons Public Domain Dedication waiver (http://creativeco mmons.org/publicdomain/zero/1.0/) applies to the data made available in this article, unless otherwise stated in a credit line to the data. 


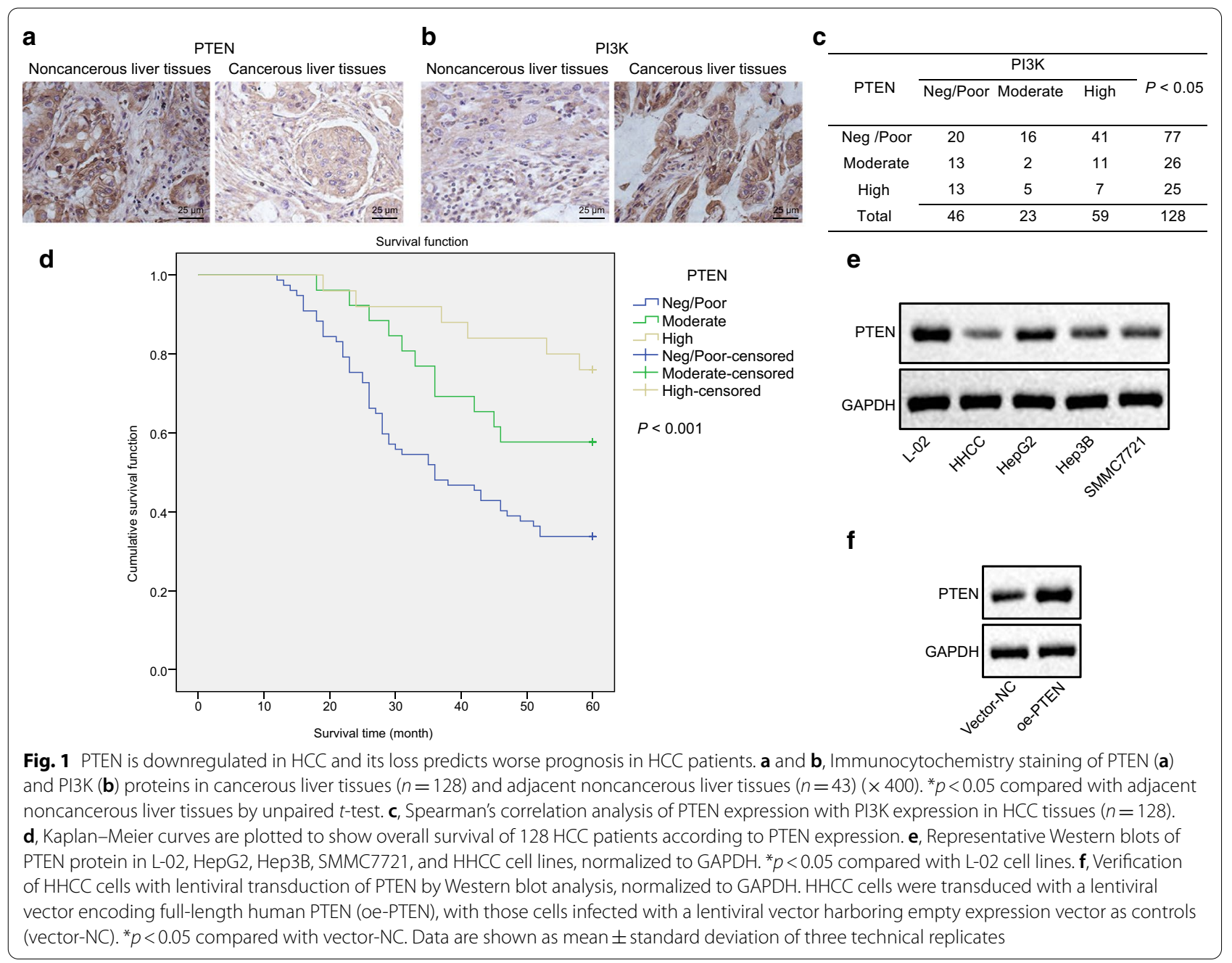




\section{a}

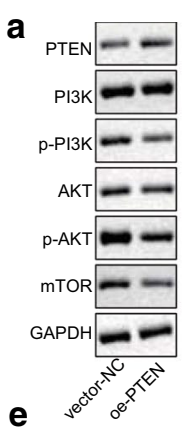

vector-NC

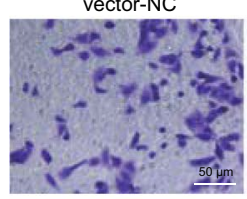

g

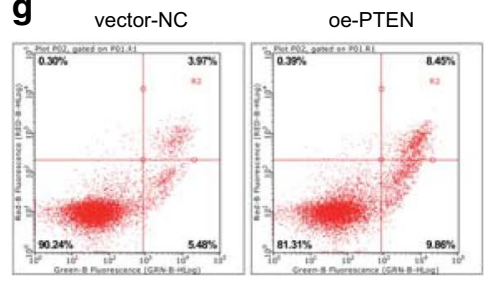

b

vector-NC

oe-PTEN

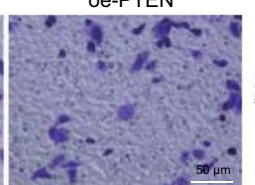

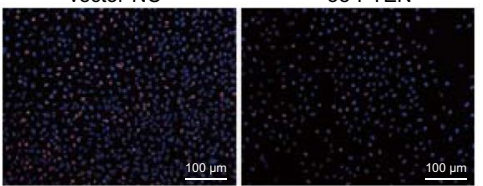

1000 min
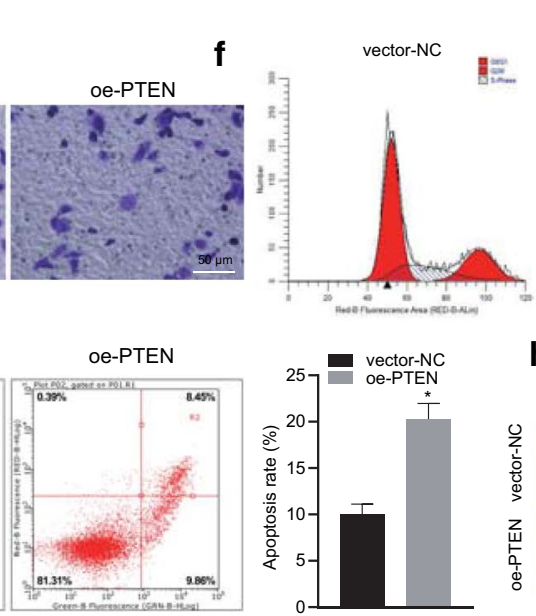

c

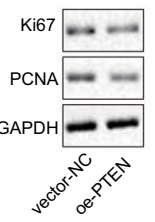

\section{d}

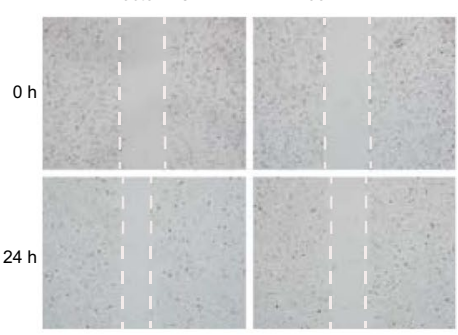

oe-PTEN
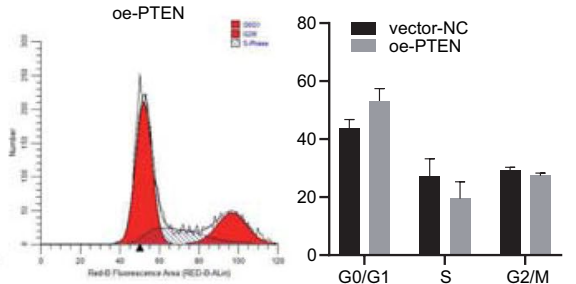

h
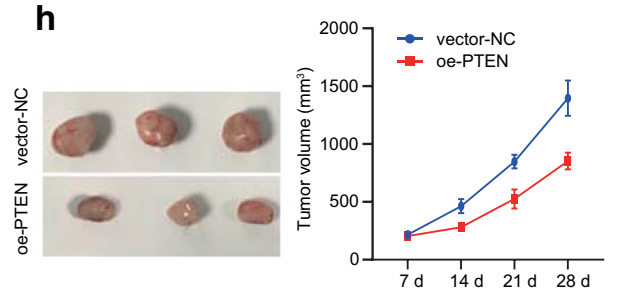

Fig. 2 PTEN regulates the development of HCC in vitro and in vivo by inhibiting the activation of PI3K/Akt pathway. HHCC cells were transduced with a lentiviral vector encoding full-length human PTEN (oe-PTEN), with those cells infected with a lentiviral vector harboring empty expression vector as controls (vector-NC). a, Representative Western blots of PI3K, Akt, and mTOR proteins and their quantitation in HHCC cells, normalized to GAPDH. b, EdU-stained cells were captured ( $\times 200)$ to reflect HHCC cell proliferation. $\mathbf{c}$, Representative Western blots of proliferation markers Ki67 and PCNA and their quantitation in HHCC cells, normalized to GAPDH. d, Wound closure was monitored to measure HHCC cell migration ( $24 \mathrm{~h}$ after scratch). e, HHCC cells invading from Matrigel-coated the upper transwell chamber into the lower one. f, Flow cytometric analysis of PI staining was performed to examine the distribution of HHCC cells throughout GO/G1, S, and G2/M phases of the cell cycle. g, Flow cytometric analysis of Annexin V/PI double staining was performed to determine HHCC cell apoptosis. $\mathbf{h}$, Representative HCC xenograft tumors and the growth of HCC xenograft tumor measured every 7 days in nude mice $(n=6) .{ }^{*} p<0.05$ compared with vector-NC by unpaired $t$-test. Data are shown as mean \pm standard deviation of three technical replicates 


\section{a}
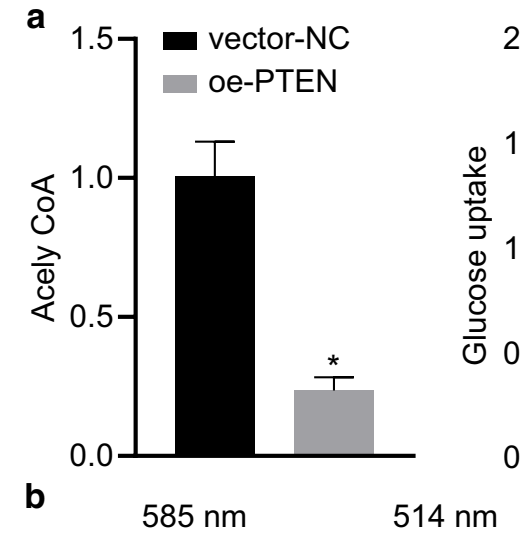$$
\text { b }
$$
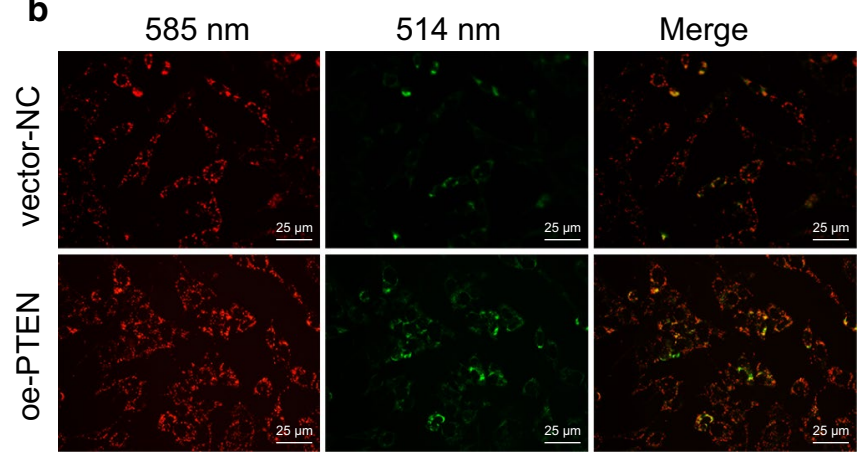

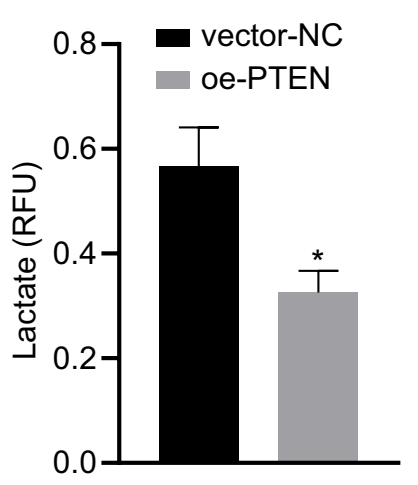

c

Merge

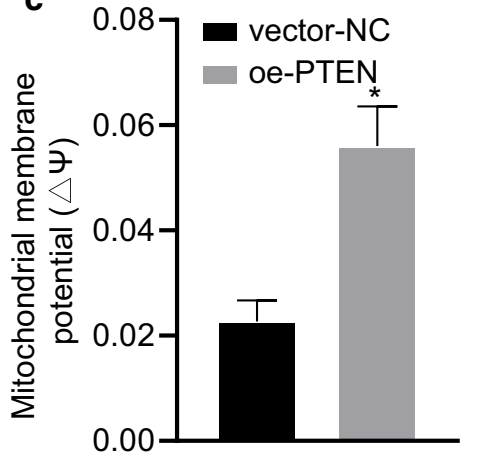

Fig. 3 PTEN inhibits Warburg effect and maintains mitochondrial function in HCC cells. HHCC cells were transduced with a lentiviral vector encoding full-length human PTEN (oe-PTEN), with those cells infected with a lentiviral vector harboring empty expression vector as controls (vector-NC). a, Measurements of acetyl-CoA synthesis, glucose uptake, and lactate production in HHCC cells. b. JC-1 staining was performed to examine the MMP in HHCC cells $(\times 400)$. Red stains at $585 \mathrm{~nm}$ indicate JC-1 aggregates in intact mitochondria, and green stains at $514 \mathrm{~nm}$ indicate JC-1 monomer in apoptotic cells with depolarization of MMP. c, Mitochondrial membrane potential $\Delta \Psi$ was calculated by flow cytometric analysis in HHCC cells. ${ }^{*} p<0.05$ compared with vector-NC by unpaired $t$-test. Data are shown as mean \pm standard deviation of three technical replicates 

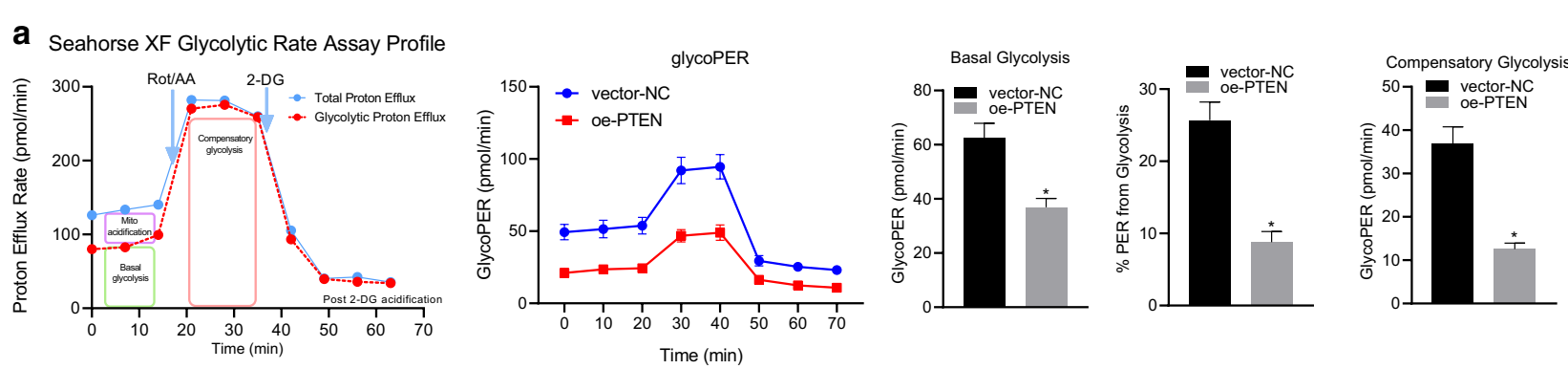

b Seahorse XF Cell Mito Stress Test Profile
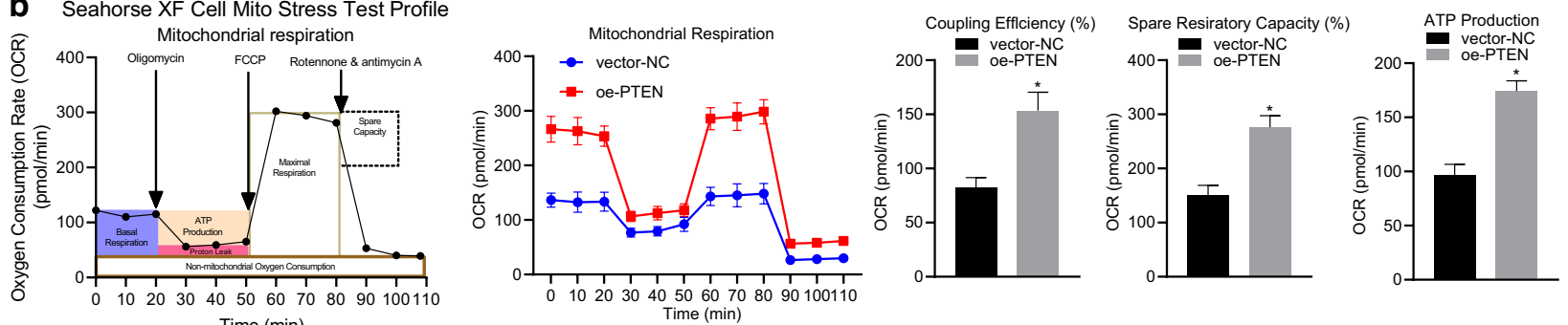

Fig. 4 PTEN reduces ECAR and enhances OCR in HCC cells. HHCC cells were transduced with a lentiviral vector encoding full-length human PTEN (oe-PTEN), with those cells infected with a lentiviral vector harboring empty expression vector as controls (vector-NC). At first, the ECAR and OCR were measured under basal condition. Next, the ECAR was measured in the presence of rotenone plus the mitochondrial complex III inhibitor antimycin A (Rote/AA) and the glycolytic inhibitor 2-DG at indicated time points. The OCR was measured in the presence of oligomycin, the reversible inhibitor of oxidative phosphorylation FCCP (p-trifluoromethoxy carbonyl cyanide phenylhydrazone), and the Rote/AA at indicated time points. OCR is expressed as pmols/minute and ECAR as $\mathrm{mpH} /$ minute. $\mathbf{a}$, ECAR under basal glycolysis, \%ECAR from basal glycolysis, and the ECAR of compensatory glycolysis were measured by Seahorse Bioscience XF24 analyzer in HHCC cells. bo, basal OCR, ATP-linked respiration, maximal OCR, and spare respiratory capacity were measured by Seahorse Bioscience XF24 analyzer in HHCC cells. Data in the upper (a and $\mathbf{b}$ ) were compared using unpaired $t$-test and in the lower ( $\mathbf{a}$ and $\mathbf{b}$ ) by repeated measures ANOVA with Bonferroni corrections. ${ }^{*} p<0.05$ compared with vector-NC. Data are shown as mean \pm standard deviation of three technical replicates

\section{Author details}

1 Department of General Surgery, Qilu Hospital of Shandong University, No. 107, Wenhua West Road, Lixia District, Jinan 250012, Shandong Province, People's Republic of China. ${ }^{2}$ Key Laboratory for Experimental Teratology of the Ministry of Education, Department of Pathology, School of Medicine, Shandong University, Jinan 250012, People's Republic of China.

Published online: 12 May 2021

\section{Reference}

1. Zhao C, Wang B, Liu E, et al. Loss of PTEN expression is associated with $\mathrm{PI} 3 \mathrm{~K}$ pathway-dependent metabolic reprogramming in hepatocellular carcinoma. Cell Commun Signal. 2020;18:131. https://doi.org/10.1186/ s12964-020-00622-w.

\section{Publisher's Note}

Springer Nature remains neutral with regard to jurisdictional claims in published maps and institutional affiliations. 